\section{Cytokines regulate postnatal hematopoietic stem cell expansion: opposing roles of thrombopoietin and LNK}

\author{
Natalija Buza-Vidas, ${ }^{1,3}$ Jennifer Antonchuk, ${ }^{1,3}$ \\ Hong Qian, ${ }^{1}$ Robert Månsson, ${ }^{1}$ Sidinh Luc, ${ }^{1}$ \\ Sasan Zandi, ${ }^{1}$ Kristina Anderson, ${ }^{1}$ \\ Satoshi Takaki, ${ }^{2}$ Jens M. Nygren, ${ }^{1}$ \\ Christina T. Jensen, ${ }^{1}$ and \\ Sten Eirik W. Jacobsen ${ }^{1,4}$ \\ ${ }^{1}$ Hematopoietic Stem Cell Laboratory, Lund Strategic \\ Research Center for Stem Cell Biology and Cell Therapy, \\ Lund University, 22184 Lund, Sweden; ${ }^{2}$ Department of \\ Community Health and Medicine, Research Institute, \\ International Medical Center of Japan, 162-8655 Shinjuku-ku, \\ Tokyo, Japan
}

The role of cytokines as regulators of hematopoietic stem cell (HSC) expansion remains elusive. Herein, we identify thrombopoietin (THPO) and the cytokine signaling inhibitor LNK, as opposing physiological regulators of HSC expansion. Lnk $^{-/-}$HSCs continue to expand postnatally, up to 24 -fold above normal by 6 mo of age. Within the stem cell compartment, this expansion is highly selective for self-renewing long-term HSCs (LTHSCs), which show enhanced THPO responsiveness. $\mathrm{Lnk}^{-/-}$HSC expansion is dependent on THPO, and 12wk-old $\mathrm{Lnk}^{-/-} \mathrm{Thpo}^{-/-}$mice have 65-fold fewer LT-HSCs than $\mathrm{Lnk}^{-/-}$mice. Expansions of multiple myeloid, but not lymphoid, progenitors in $\mathrm{Lnk}^{-/-}$mice also proved THPO-dependent.

Supplemental material is available at http://www.genesdev.org.

Received March 3, 2006; revised version accepted May 19, 2006.

As self-renewing hematopoietic stem cells (HSCs) are not only a requisite for sustained normal blood cell production but also of critical importance for rapid and sustained reconstitution after bone marrow (BM) transplantation, identification and clinical exploitation of the stem cell self-renewal machinery have been a longsought goal of HSC research (Sorrentino 2004). HSCs cycle and expand extensively during fetal development and following transplantation (Pawliuk et al. 1996; Iscove and Nawa 1997), providing compelling evidence for a positive and dynamic regulation of HSC self-renewal. However, from early postnatal life the HSC compartment is sustained at a relatively constant level (Morrison et al. 1996). This tight restriction on postnatal HSC ex-

[Keywords: LNK; stem cells; self-renewal; hematopoiesis; thrombopoietin]

${ }^{3}$ These authors contributed equally to the work.

${ }^{4}$ Corresponding author.

E-MAIL sten.jacobsen@med.lu.se; FAX 46-46-2223600.

Article is online at http://www.genesdev.org/cgi/doi/10.1101/gad.385606. pansion, potentially reflecting the enhanced propensity of HSCs to undergo leukemic transformation (Reya et al. 2001), implicates either the coexistence of important negative regulators of HSC self-renewal, or down-regulation of positive regulators in steady-state hematopoiesis.

Although HSCs represent the best characterized somatic stem cells, the physiological regulators of HSC self-renewal and expansion remain largely unknown. However, a few intrinsic cues-including the transcriptional repressor BMI-1 (Molofsky et al. 2003; Iwama et al. 2004), the proto-oncogene MYC (Wilson et al. 2004), and the transcription factor $\mathrm{C} / \mathrm{EBP} \alpha$ (Zhang et al. 2004)have, through lack-of-function studies, been implicated as regulators of HSC self-renewal. Further, overexpression of HoxB4 (Antonchuk et al. 2002) promotes extensive HSC expansion ex vivo, although evidence for its physiological role in HSC regulation is lacking.

Whereas the important role of hematopoietic cytokines as extracellular regulators of blood lineage development is indisputably established (Ogawa 1993), efforts to identify their expected roles as positive regulators of HSC self-renewal and expansion have been disappointing (Sauvageau et al. 2004; Sorrentino 2004; Jacobsen 2005). Despite more than two decades of exhaustive attempts to promote ex vivo HSC expansion using HSCstimulating cytokines, most efforts have rather resulted in HSC loss, and only in a few cases has limited HSC expansion been achieved (Miller and Eaves 1997; Zhang and Lodish 2005). Consequently, the enthusiasm to utilize cytokines for HSC ex vivo expansion has waned, and whether cytokines play any role in regulating HSC expansion under physiological conditions has increasingly been questioned (Sauvageau et al. 2004; Jacobsen 2005). In fact, of all cytokines investigated, only genetic deficiencies in Thrombopoietin (Thpo) or its receptor $M p l$ result in reduced levels of HSCs (Kimura et al. 1998; Solar et al. 1998). As THPO efficiently suppresses apoptosis (Pestina et al. 2001) and promotes survival of HSCs (Borge et al. 1996), it has been suggested that THPO may act primarily to counteract HSC apoptosis, rather than promoting HSC expansion.

Recently, mice deficient in expression of $L n k$, a member of the APS family of adaptor proteins (Yokouchi et al. 1997), were demonstrated to have a variety of hematopoietic phenotypes, including an expansion of the HSC compartment (Takaki et al. 2000; Ema et al. 2005). However, the dynamics of this expansion and the mechanism of action by which LNK might restrict HSC expansion in steady-state hematopoiesis remain unclear, as LNK acts as a broad inhibitor of growth factor and cytokine signaling pathways, including those of THPO, Kit ligand (KITL), Erythropoietin (EPO), Interleukin (IL)-3, and IL-7 (Takaki et al. 2000, 2002; Velazquez et al. 2002; Tong and Lodish 2004; Tong et al. 2005).

The present studies were designed to identify whether LNK might act as a physiological negative regulator of cytokine-induced HSC expansion, and if so what specific cytokine signaling pathway it might directly target. Herein we provide compelling evidence for LNK being a physiological negative regulator of THPO-induced postnatal expansion of self-renewing HSCs and myeloid progenitors, identifying a unique balance between opposing 
regulators of cytokine receptor signaling in regulating the size of the HSC and progenitor compartments.

\section{Results and Discussion}

Selective and age-dependent expansion of long-term HSCs in $\mathrm{Lnk}^{-1-}$ mice

Although previous studies had established that the HSC compartment is expanded in adult $\mathrm{Lnk}^{-/-}$mice (Takaki et al. 2002; Ema et al. 2005), the role of LNK in regulating recently identified phenotypically and functionally distinct subclasses of HSCs (Adolfsson et al. 2005; Yang et al. 2005) had not been investigated.

Notably, whereas previous studies had suggested that $\mathrm{Lnk}^{-1-}$ HSCs, defined as $\mathrm{LIN}^{-} \mathrm{SCA} 1^{+} \mathrm{KIT}^{+} \mathrm{CD} 34^{-}$ $\left(\mathrm{LSKCD}^{-} 4^{-}\right)$, are not expanded relative to wild-type HSCs prior to the age of $8 \mathrm{wk}$ (Ema et al. 2005), we observed a 1.9-fold $(p<0.01)$ increase in $\mathrm{Lnk}^{-}$ LSKFLT3 $^{-}$(LSKFLT3 ${ }^{-}$) cells already in $14.5 \mathrm{~d}$ postcoitum (dpc) fetal liver (FL) (Fig. 1A,B). The seeming discrepancy between our finding of expanded LSKFLT3-
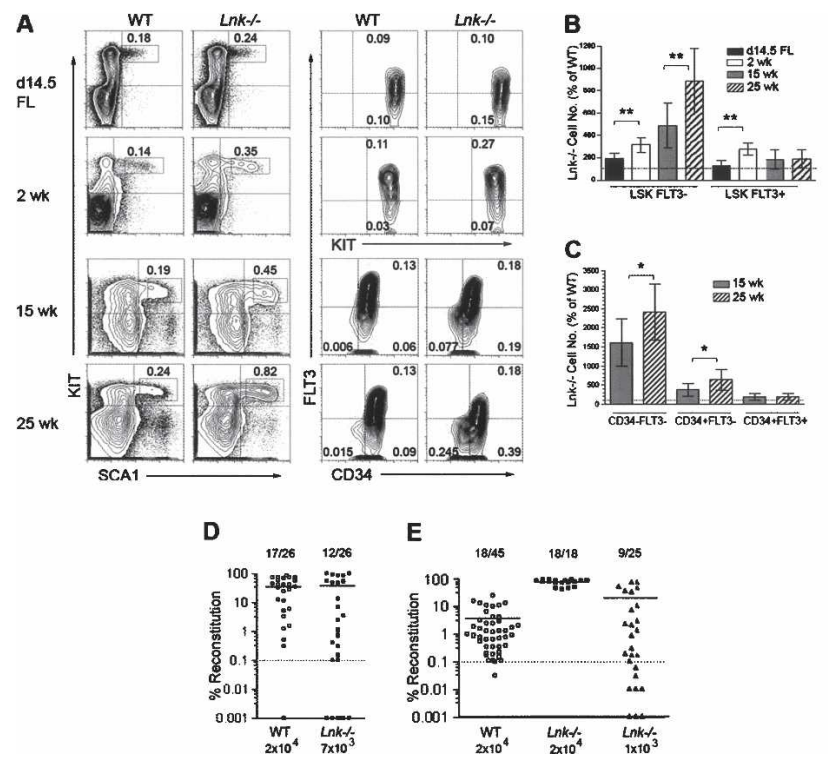

Figure 1. Extensive age-dependent expansion of LSKCD34-FLT3LT-HSCs in $\mathrm{Lnk}^{-1-}$ mice. (A) Representative FACS profiles of SCA1and KIT-expressing cells within the LIN $^{-}$population, showing LSK gating (left panels), and CD34 and FLT3 expression within the LSK population (right panels), from 14.5-dpc FL and 2- to 25-wk-old BM from wild-type (WT) and $\mathrm{Lnk}^{-/-}$mice. Numbers shown are mean percentages of total cells in four to 12 mice of each genotype. $(B)$ Mean $\left.{ }_{ \pm} \mathrm{SD}\right)$ numbers of LSKFLT3 ${ }^{-}$and LSKFLT3 $^{+}$subpopulations from day 14.5 FL $(n=7-8)$, and 2-wk-old $(n=4), 15$-wk-old $(n=12)$, or 25-wk-old $(n=7) \mathrm{BM}$ cells in $\mathrm{Lnk}^{-1-}$ mice relative to age-matched wild-type (WT) controls. $(C)$ Mean $( \pm \mathrm{SD})$ numbers of LSKCD34-FLT3 $^{-}$, LSKCD34 ${ }^{+} \mathrm{FLT3}^{-}$, and LSKCD34 $4^{+} \mathrm{FLT}^{+}{ }^{+}$cells in BM of 15-wk-old $(n=12)$ and 25-wk-old $(n=7) \mathrm{Lnk}^{-/-}$mice relative to age-matched wild-type (WT) controls. Cellularities were increased 1.2- to 1.4-fold in $\mathrm{Lnk}^{-/-}$mice. $(D, E)$ Total PB reconstitution by donor-derived cells at $16 \mathrm{wk}$ after transplantation of limiting doses of wild-type (WT) or $\mathrm{Lnk}^{-/-}$14.5-dpc FL cells $(D)$ and 16-wkold BM cells $(E)$. Each symbol represents an individual recipient and bars indicate mean reconstitution in positive mice. Fractions shown above are the numbers of mice positive for lympho-myeloid reconstitution, using the threshold level of $0.1 \%$ total cells (dashed line), and $0.02 \%$ of each of the $\mathrm{B}, \mathrm{T}$, and myeloid lineages. $\left(^{\star}\right) p<0.05 ;\left(^{\star \star}\right)$ $p<0.01$. cells and no expansion of LSKCD34- cells in early development reported by Ema et al. (2005) is most likely explained by HSCs not having a LSKCD34- but rather a LSKCD $34^{+}$phenotype in early development (Ito et al. 2000), whereas HSCs are LSKFLT3- throughout development (Adolfsson et al. 2001; Christensen and Weissman 2001; L. Yang and S.E.W. Jacobsen, unpubl.). Thus, in contrast to previously published findings, our data suggest that LNK is already a negative regulator of the extensive physiological HSC expansion that takes place during this stage of fetal development (Pawliuk et al. 1996). Importantly, the expansion of $\mathrm{Lnk}^{-/-}$LSKFLT3 $^{-}$ cells gradually increased with age, so that the numbers of $\mathrm{Lnk}^{-1}$ LSKFLT3 $^{-}$cells were increased relative to wildtype mice by 3.2-fold, 4.8-fold, and 8.8-fold in 2-wk-old, 15-wk-old, and 25-wk-old mice, respectively (Fig. 1A,B). Whereas LSKFLT3 $^{-}$cells contain pluripotent HSCs, LSKFLT3 $^{+}$cells are predominantly lymphoid-primed multipotent progenitors (LMPPs) (Adolfsson et al. 2005). In striking contrast to the large expansions of LSKFLT3 cells, the LSKFLT3 ${ }^{+}$compartment was only increased 1.3- to 2.7-fold in FL or adult BM (Fig. 1B).

In adult mice, costaining for CD34 and FLT3 expression allows further discrimination between LSKCD34-FLT3long-term HSCs (LT-HSCs) and LSKCD34 ${ }^{+} \mathrm{FLT}^{-}$shortterm HSCs (ST-HSCs) (Ito et al. 2000; Yang et al. 2005). Our analyses demonstrated dramatic 16-fold and 24-fold increases in LSKCD34-FLT3 ${ }^{-}$cells in 15-wk-old and 25wk-old $\mathrm{Lnk}^{-1-}$ mice, respectively (Fig. 1C). Conversely, LSKCD $34^{+} \mathrm{FLT}^{-}$cells were increased much less (4.1fold and 6.3-fold, $p<0.001$ and 0.01 , respectively, compared with wild type). As shown in wild-type mice (Yang et al. 2005), we also found that all long-term multilineage repopulating activity was restricted to the LSKCD34-FLT3- compartment in adult $\mathrm{Lnk}^{-/-}$mice (N. Buza-Vidas and S.E.W. Jacobsen, unpubl.).

We next sought to verify the expansion of LT-HSCs by functional assessment. Thus, limiting dose long-term competitive transplantation assays were performed with wild-type and $\mathrm{Lnk}^{-/}$FL cells or adult BM cells, along with a standard competitor dose of $2 \times 10^{5} \mathrm{BM}$ cells (Yang et al. 2005). In recipients of FL cells, 17 of 26 (65\%) recipients of 20,000 wild-type cells and 12 of $26(46 \%)$ recipients of $7000 \mathrm{Lnk}^{-/}$cells showed sustained multilineage reconstitution (Fig. 1D). This translates into a 1.7-fold increase in the frequency of LT-HSCs in $\mathrm{Lnk}^{-/-}$ FLs (see Supplemental Material), in agreement with the observed 1.9-fold increase in LSKFLT3 ${ }^{-}$FL cells (Fig. 1B).

Similarly, based on the frequency of multilineage reconstituted mice transplanted with 20,000 wild-type or $1000 \mathrm{Lnk}^{-/-}$adult BM cells (Fig. 1E), a nearly 17-fold increase in the frequency of LT-HSCs was calculated for 16-wk-old $\mathrm{Lnk}^{-/-}$mice, in agreement with the 16-fold increase in $\mathrm{LSKCD}^{-} 4^{-} \mathrm{FLT}^{-}$cells at that age (Fig. 1C). The relative contribution of $\mathrm{Lnk}^{-1-}$ HSCs toward B-cell reconstitution was slightly enhanced and $\mathrm{T}$ cells were reduced, whereas myeloid contribution was comparable with that from wild-type cells (Supplementary Fig. 1A). Secondary transplantation of BM cells from positively reconstituted primary recipients of limiting dose transplantations (FL or BM) demonstrated that postnatally expanded $L n k^{-/-}$HSCs sustain self-renewal ability, at least comparable with that of age-matched wild-type HSCs, as the frequency of multilineage reconstituted secondary recipients of $\mathrm{Lnk}^{-1-}$ cells was slightly increased when compared with that of wild-type cells (Supplementary 
Buza-Vidas et al.

Fig. 1B,C), in agreement with previous studies (Ema et al. 2005). However, this enhanced potential for serial reconstitution appears rather limited when compared with the extensive HSC expansion in $\mathrm{Lnk}^{-/-}$mice observed postnatally, when expansion of HSCs is normally restricted. This, together with the rather limited increase in fetal expansion of HSCs in $\mathrm{Lnk}^{-/-}$mice, suggests that the role of LNK might primarily be to limit HSC expansion during steady-state hematopoiesis, and that other, LNK-independent, mechanisms might be primarily responsible for promoting the extensive HSC expansion observed during fetal development and post-transplantation (Pawliuk et al. 1996).

At variance with previous studies (Ema et al. 2005), BrdU incorporation experiments suggested that the cycling of adult LT-HSCs is not enhanced in $\mathrm{Lnk}^{-/-}$mice (Supplementary Fig. 2). Interestingly, this suggests that the ability of LNK to suppress postnatal expansion of HSCs is not coupled to regulation over the extent of HSC proliferation. Importantly, leukemia was never observed in $\mathrm{Lnk}^{-/-}$mice or in primary or secondary recipients of $\mathrm{Lnk}^{-/-} \mathrm{BM}$ cells (N. Buza-Vidas, J. Antonchuk, and S.E.W. Jacobsen, unpubl.).

Thus, $\mathrm{Lnk}^{-/-}$mice have a selective and age-progressive expansion of the minor LT-HSC compartment, identifying LNK as a unique negative regulator selectively targeting LT-HSCs.

\section{Expansion of LT-HSCs in $\mathrm{Lnk}^{-/-}$mice is dependent on thrombopoietin signaling}

LNK has been demonstrated to directly inhibit a number of signaling pathways (Takaki et al. 2002; Velazquez et al. 2002; Tong and Lodish 2004; Tong et al. 2005), and of these the KIT and THPO pathways have been implicated as potential regulators of HSC numbers and/or function (Miller et al. 1996; Kimura et al. 1998; Solar et al. 1998). As previous studies had failed to couple increased HSC numbers in $\mathrm{Lnk}^{-/-}$mice to enhanced KIT function (Takaki et al. 2002; Ema et al. 2005), we sought here to establish whether LNK-induced inhibition of HSC expansion might depend on THPO signaling.

Whereas the expression of Bmi-1 and HoxB4 as determined by Q-PCR analysis was virtually unaffected in $\mathrm{Lnk}^{-/-}$LSKCD 34 $^{-} \mathrm{FLT3}^{-}$cells, Mpl expression was slightly increased (1.6-fold) (Fig. 2A). Notably, the in vitro THPO proliferative responsiveness of single $\mathrm{Lnk}^{-/-}$ LSKCD 34 $^{-}$FLT3 $^{-}$cells was enhanced both with regard to the number $(2.5$-fold) and size $(6.3$-fold) of the clones formed (Fig. 2B). Furthermore, LSKCD $34^{+}$FLT3 $^{-}$ (ST-HSCs) were less responsive to THPO overall, their THPO responsiveness was only marginally enhanced in the Lnk-deficient setting, and neither wild-type nor $\mathrm{Lnk}^{-/-} \mathrm{LSKCD}^{+} 4^{+} \mathrm{FLT}^{\mathrm{hi}}$ (LMPPs) responded to THPO (Fig. 2B). These results are in agreement with the selective expansion of LT-HSCs in $\mathrm{Lnk}^{-/-}$mice, potentially via its interaction with THPO signaling. In further support of this, $M p 1$ and Lnk expression were highest in LSKCD34-FLT3cells, and much lower in LSKCD $34^{+}$FLT3 ${ }^{\text {hi }}$ cells (Fig. 2C).

The above findings, combined with the documented ability of LNK to directly inhibit THPO-induced signaling (Tong and Lodish 2004), suggested a model in which expansion of LT-HSCs in $\mathrm{Lnk}^{-1-}$ mice involves THPO signaling. Thus, we next studied the requirement of THPO in expansion of $\mathrm{Lnk}^{-/-} \mathrm{HSCs}$ by examining the HSC compartment in mice lacking both Lnk and Tpo
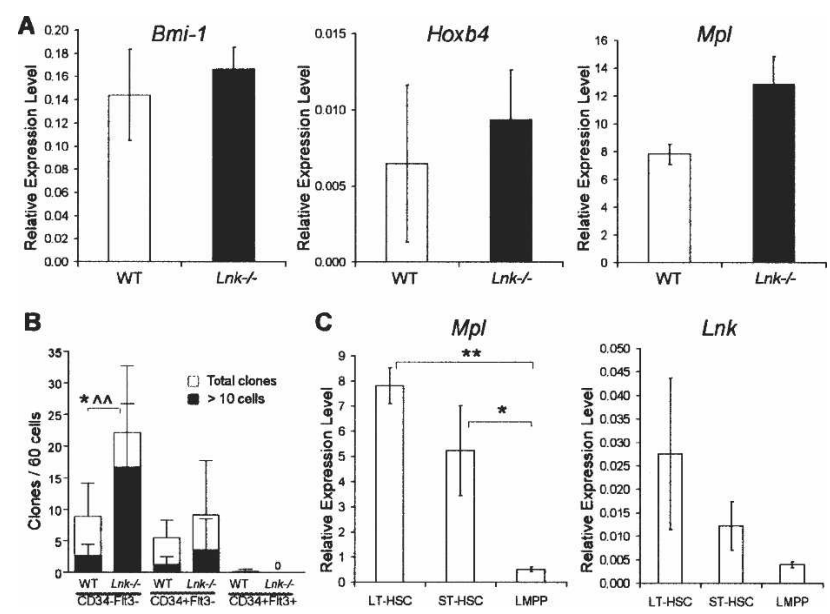

Figure 2. Enhanced proliferative responsiveness of $\mathrm{Lnk}^{-/-} \mathrm{HSCs}$ to thrombopoietin. (A) Bmi-1, Hoxb4, and Mpl mRNA expression levels in LSKCD34-FLT3- cells from 12-wk-old wild-type (WT) and $\mathrm{Lnk}^{-/-}$mice, as determined by Q-PCR. Mean ( \pm SEM) expression levels, relative to HPRT, from two to three experiments. (B) Mean $( \pm \mathrm{SD})$ number of proliferative clones per 60 single LSKCD34 ${ }^{-} \mathrm{FLT}^{-}{ }^{-}$, $\mathrm{LSKCD}_{34}{ }^{+} \mathrm{FLT}^{-}{ }^{-}$, or $\mathrm{LSKCD}_{3}{ }^{+} \mathrm{FLT}^{+}$cells seeded in $50 \mathrm{ng} / \mathrm{mL}$ THPO. Open bars indicate mean number of total clones, closed bars indicate number of clones $>10$ cells, from three experiments. $\left.{ }^{*}\right)$ $p<0.05$ total number of clones; $\left({ }^{\wedge}\right) p<0.01$ number of large clones. (C) Mpl and Lnk mRNA expression levels in 2000 wild-type

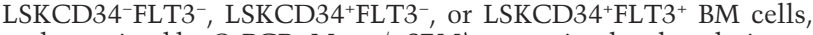
as determined by Q-PCR. Mean $\left({ }_{ \pm}\right.$SEM $)$expression levels, relative to HPRT, from three to five experiments. $\left(^{\star}\right) p<0.05 ;\left(^{\star \star}\right) p<0.01$.

expression. Strikingly, the extensive expansion of LSKCD 34-FLT3- cells in Lnk $^{-1-}$ as compared with wildtype mice (17-fold) could not be observed when comparing 12-wk-old $\mathrm{Thpo}^{-1-}$ and $\mathrm{Lnk}^{-/-} \mathrm{Thpo}^{-/-}$mice (Fig. $3 \mathrm{~A}, \mathrm{~B})$. In fact, the number of LSKCD34-FLT3- cells were reduced by $88 \%(p<0.001)$ in $\mathrm{Thpo}^{-1-}$ mice and by $82 \%$ in $\mathrm{Lnk}^{-1-} \mathrm{ThpO}^{-/-}$mice, giving a 100 -fold difference in $\mathrm{LSKCD}^{-} 4^{-} \mathrm{FLT3}^{-}$numbers between $\mathrm{Lnk}^{-/}$and $\mathrm{Lnk}^{-/-}$ Thpo $^{-1-}$ mice (Fig. 3A,B).

Limiting dilution transplantation experiments-where positively reconstituted mice were defined as having at least $0.1 \%$ donor-derived total blood cells and $0.02 \% \mathrm{my}$ eloid, B-, and T-lymphoid donor-derived cells at $4 \mathrm{mo}$ post-transplant (Materials and Methods)-were in agreement with the LSKCD $34^{-} \mathrm{FLT3}^{-}$analysis, in that longterm competitive repopulating units (CRUs) were increased as much as 25 -fold in $\mathrm{Lnk}^{-/-}$relative to wild-type mice $(p<0.001)$ and extensively decreased in Thpo ${ }^{-/-}$ mice (18-fold, $p<0.001)$. $\mathrm{Lnk}^{-1-} \mathrm{Thpo}^{-/-}$mice had CRU values sevenfold above that in Thpo ${ }^{-/-}$mice $(p=0.0038)$, but still significantly lower than wild-type mice $(p=0.0078)$, giving a 65-fold difference in CRUs between $\mathrm{Lnk}^{-/-}$and $\mathrm{Lnk}^{-/-} \mathrm{Thpo}^{-/-}$mice. Similar differences were observed when setting the threshold values for total and lineage reconstitution at a higher level $(1.0 \%$ and $0.05 \%$, respectively) (Supplementary Fig. 3).

Thus, the phenotypic and functional evaluations of HSC numbers demonstrate that the expansion of HSCs in $\mathrm{Lnk}^{-/-}$mice is largely THPO-dependent, supporting that LNK restricts LT-HSC expansion at least in part through inhibiting THPO-stimulated HSC expansion.

The expansion of LSKCD $34^{+}$FLT3 $^{-}$ST-HSCs due to Lnk deficiency was also reduced when comparing Thpo $^{-/-}$and $\mathrm{Lnk}^{-/-} \mathrm{Thpo}^{-/-}$mice (Fig. 3A,B). Conversely, the slight (twofold, $p<0.01$ ) expansion of LSKCD $34^{+}$FLT3 $^{+}$ 


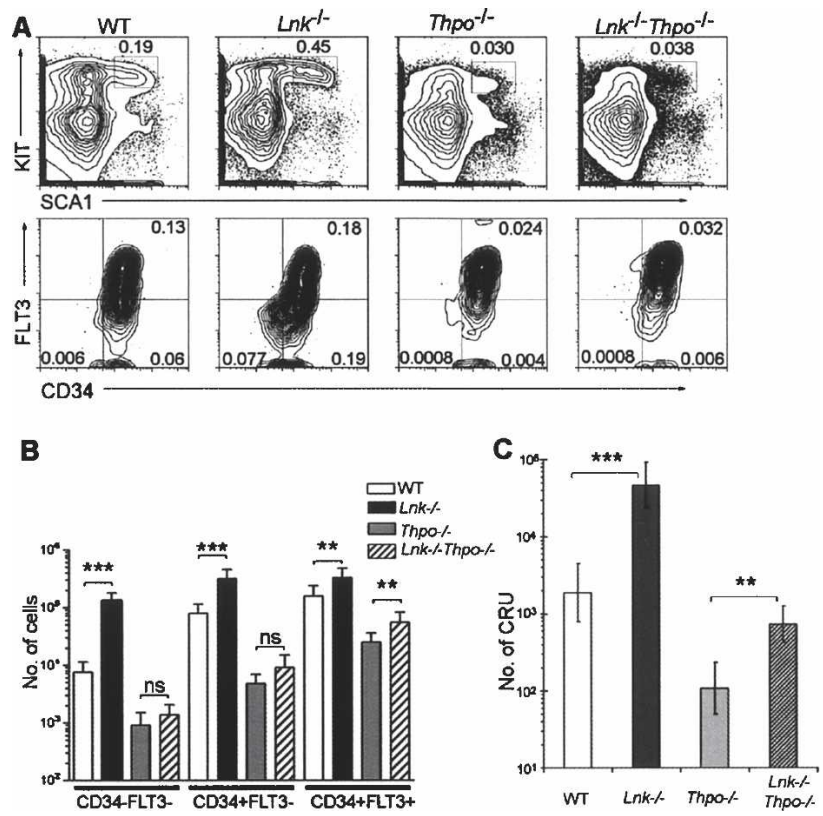

Figure 3. HSC expansion in Lnk-deficient mice is dependent on THPO. (A) Representative FACS profiles of SCA1- and KIT-expressing cells within the $\mathrm{LIN}^{-}$population, showing LSK gating (top panels), and CD34 and FLT3 expression within the LSK population (bottom panels), in BM from 12-wk-old wild-type (WT), $\mathrm{Lnk}^{-1}$, $\mathrm{Thpo}^{-/-}$, or $\mathrm{Lnk}^{-/-} \mathrm{Thpo}^{-/-}$mice ( $\mathrm{n}=5$ each). Numbers shown are mean percentages of total cells in each subpopulation. (B) Mean

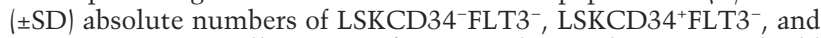
LSKCD $34^{+} \mathrm{FLT}^{+}$cells per two femurs and two tibias in 12 -wk-old mice ( $n=5$ per genotype). (C) CRU numbers $( \pm 95 \%$ confidence intervals) in two femurs and two tibias of BM from wild-type (WT), $\mathrm{Lnk}^{-/-}$, Thpo- ${ }^{-/}$, and $\mathrm{Lnk}^{-/-} \mathrm{Thpo}^{-/-} 12$-wk-old mice, averaged from two replicate experiments. $\left(^{\star}\right) p<0.05 ;\left(^{\star \star}\right) p<0.01 ;\left(^{\star \star \star}\right) p<0.001$; (ns) not significant.

cells in $\mathrm{Lnk}^{-/-}$mice did not appear to involve THPO signaling (Fig. 3A,B), in agreement with the inability of LMPPs to respond to THPO, and implicating LNK targeting of other pathways in LMPPs.

The expression of Lnk in HSCs, the enhanced THPO responsiveness of purified $\mathrm{Lnk}^{-1-} \mathrm{HSCs}$, and the HSC phenotype in $\mathrm{Lnk}^{-/-}$mice reflecting the restricted pattern of MPL expression on HSCs all support a cell-autonomous increase in HSC numbers in $\mathrm{Lnk}^{-/-}$mice. To further investigate this, we compared reconstitution levels of wild-type BM cells transplanted into lethally irradiated wild-type and $\mathrm{Lnk}^{-/-}$recipients (Supplementary Fig. 4). As long-term multilineage reconstitution was in fact slightly lower in $\mathrm{Lnk}^{-1-}$ than wild-type recipients, these findings support that the expansion of HSCs in $\mathrm{Lnk}^{-/-}$mice is due to an intrinsic loss of LNK function in LT-HSCs.

\section{LNK inhibits THPO-dependent expansion of colony- forming unit-spleen (CFU-S) and myelo-erythroid progenitors}

We next explored to what degree the reported increases of myeloid and B lymphoid progenitors in $\mathrm{Lnk}^{-1-}$ mice (Velazquez et al. 2002) and corresponding decrease of myeloid progenitors in $\mathrm{Thpo}^{-/-}$mice (Carver-Moore et al. 1996) could also reflect LNK-mediated inhibition of THPO-dependent expansion of committed myeloid progenitors.
The CFU-S assay detects multipotent myelo-erythroid progenitors with limited self-renewal ability (Till and McCulloch 1961). Adult $\mathrm{Lnk}^{-/-}$mice had a 2.6-fold expansion of day 12 CFU-S, whereas these were decreased to $18 \%$ of wild type in $\mathrm{Thpo}^{-/-}$mice (Fig. 4A). As CFU-S numbers were comparable in $\mathrm{Thpo}^{-/-}$and $\mathrm{Lnk}^{-/-} \mathrm{Thpo}^{-/-}$ mice, the CFU-S expansion observed in $\mathrm{Lnk}^{-/}$mice also proved to be strictly dependent on THPO signaling.

Committed granulocyte-macrophage (CFU-GM) (Fig. 4B), erythroid (BFU-E) (Fig. 4C), and megakaryocyte (CFU-Mk) (Fig. 4D) progenitors were all increased in $\mathrm{Lnk}^{-/-}$mice and reduced in $\mathrm{Thpo}^{-/-}$mice, and, similar to CFU-S, their increase in $\mathrm{Lnk}^{-/-}$mice was entirely THPO-dependent.

In agreement with previous studies (Takaki et al. 2000; Velazquez et al. 2002), we also found early B220+ $\mathrm{IgM}^{-} \mathrm{B}$ cells to be expanded in the BM of $\mathrm{Lnk}^{-/}$mice, but, in contrast to myeloid progenitors, this expansion was entirely THPO-independent (Fig. 4E), in agreement with B cells being unaffected in Thpo-/- mice (de Sauvage et al. 1996). The coupling of LNK and THPO as opposing regulators of myeloid but not lymphoid lineage development was also evident in peripheral blood (PB) (Table 1), as the increase in PB lymphocytes in $\mathrm{Lnk}^{-/}$mice was THPOindependent, whereas the increases in neutrophils, monocytes, eosinophils, and platelets were all THPOdependent.

In conclusion, these studies demonstrate that $\mathrm{Lnk}^{-/-}$ mice have progressive and selective increases in LTHSCs and myeloid progenitors that are dependent on THPO signaling. The idea that LNK and THPO might function as interacting but opposing physiological regulators of HSC expansion is strongly supported by several lines of evidence. First, signaling studies of $\mathrm{Lnk}^{-/-}$cells demonstrate that LNK is a direct inhibitor of THPOinduced signaling (Tong and Lodish 2004), and in agreement with this we demonstrate here that $\mathrm{Lnk}^{-/-}$
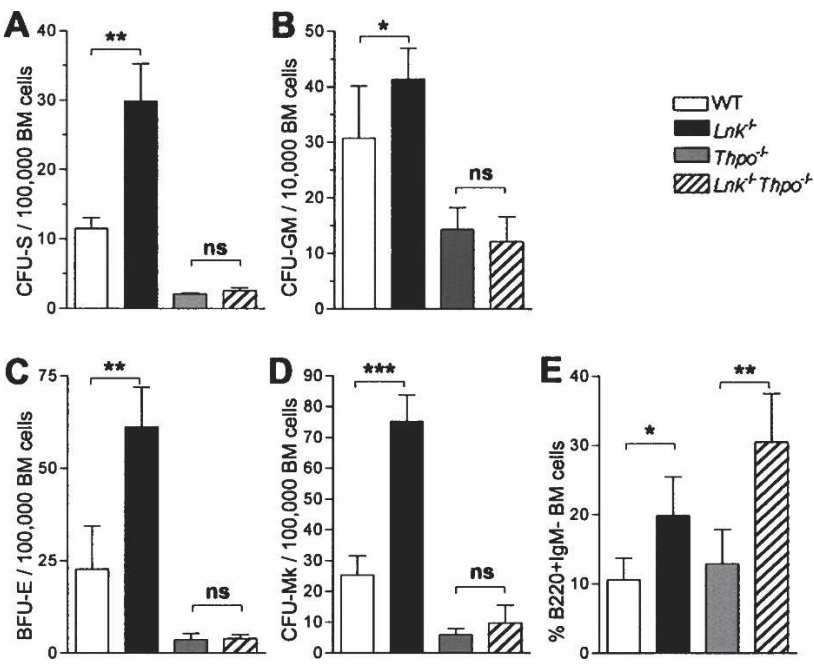

Figure 4. THPO-dependent expansion of CFU-S and myelo-erythroid progenitors in $\mathrm{Lnk}^{-/-}$mice. $(A-D)$ Mean $( \pm \mathrm{SD})$ numbers in 12-wk-old adult BM of wild-type (WT), $\mathrm{Lnk}^{-/-}, \mathrm{ThpO}^{-/-}$, and $\mathrm{Lnk}^{-/} \mathrm{Thpo}^{-/-}$mice of day 12 CFU-S per 100,000 BM cells $(n=3$ per genotype, 4 recipients each) $(A)$; CFU-GM per 10,000 cells plated $(n=6-7$ each $)(B)$; BFU-E per 100,000 cells plated $(n=4-5$ each $)(C)$; CFU-Mk per 100,000 cells plated $(n=4-5$ each $)(D)$. $(E)$ Mean $( \pm$ SD $)$ percentage of BM B220+IgM ${ }^{-}$cells $(n=5-6) .\left(^{*}\right) p<0.05 ;\left(^{* *}\right) p<0.01$; $\left.{ }^{\star \star \star *}\right) p<0.001 ;($ ns) not significant. 
Table 1. THPO-dependent expansion of myeloid but not lymphoid lineages in Lnk-deficient mice

\begin{tabular}{|c|c|c|c|c|}
\hline & Wild type & $\operatorname{Lnk}^{-/-}$ & $\mathrm{Thpo}^{-/-}$ & $\mathrm{Lnk}^{-/-} \mathrm{Thpo}^{-/-}$ \\
\hline Red cell count $\left(\times 10^{9} / \mathrm{mL}\right)$ & $10.1( \pm 0.39)$ & $9.3( \pm 0.57)^{\mathrm{b}}$ & $9.2( \pm 0.68)$ & $9.5( \pm 1.04)$ \\
\hline Hematocrit $(\%)$ & $52.9( \pm 2.3)$ & $51.3( \pm 3.8)$ & $49.8( \pm 3.7)$ & $51.4( \pm 5.1)$ \\
\hline White cell count $\left(\times 10^{6} / \mathrm{mL}\right)$ & $7.0( \pm 1.5)$ & $30.9( \pm 4.9)^{\mathrm{c}}$ & $5.5( \pm 1.7)$ & $13.7( \pm 3.8)^{\mathrm{e}}$ \\
\hline Lymphocytes & $5.2( \pm 0.6)$ & $26.7( \pm 3.1)^{\mathrm{a}}$ & $3.6( \pm 1.6)$ & $10.3( \pm 1.6)^{\mathrm{d}}$ \\
\hline Neutrophils & $0.77( \pm 0.61)$ & $5.07( \pm 1.5)^{\mathrm{a}}$ & $0.92( \pm 0.41)$ & $1.27( \pm 0.76)$ \\
\hline Monocytes & $0.17( \pm 0.04)$ & $1.18( \pm 0.97)^{\mathrm{a}}$ & $0.11( \pm 0.06)$ & $0.33( \pm 0.28)$ \\
\hline Eosinophils & $0.14( \pm 0.11)$ & $0.59( \pm 0.18)^{\mathrm{a}}$ & $0.08( \pm 0.07)$ & $0.23( \pm 0.20)$ \\
\hline Platelet count $\left(\times 10^{6} / \mathrm{mL}\right)$ & $845( \pm 335)$ & $1391(377)^{\mathrm{b}}$ & $52( \pm 28)$ & $135( \pm 145)$ \\
\hline
\end{tabular}

Data shown are mean $( \pm \mathrm{SD})$ values from four to five mice (12 wk old) per genotype.

${ }^{\mathrm{a}} p<0.05,{ }^{\mathrm{b}} p<0.01,{ }^{\mathrm{c}} p<0.001$ in $\mathrm{Lnk}^{-/-}$compared with wild type mice.

${ }^{\mathrm{d}} p<0.05,{ }^{\mathrm{e}} p<0.001$ in $\mathrm{Lnk}^{-/-} \mathrm{Thpo}^{-/-}$compared with $\mathrm{Thpo}^{-/-}$mice.

LT-HSCs are hyperresponsive to THPO. Second, the HSC and myeloid phenotypes of $\mathrm{Lnk}^{-/-}$and $\mathrm{Thpo}^{-/-}$ mice closely oppose each other, qualitatively as well as quantitatively. Third, when compared with ST-HSCs and LMPPs, LT-HSCs express higher levels of $M p l$ and Lnk, and in agreement with this, $\mathrm{Lnk}^{-/-}$LT-HSCs show the most enhanced responsiveness to THPO. Finally and most importantly, studies of $\mathrm{Lnk}^{-/-} \mathrm{Thpo}^{-/-}$mice demonstrate that the LT-HSC as well as myeloid progenitor cell expansion in $\mathrm{Lnk}^{-/-}$mice are dependent on THPO. The fact that this THPO-dependence is restricted to HSCs and myeloid progenitors on which MPL has been shown to be expressed, but not on LMPPs or lymphoid progenitors that fail to respond to THPO, confirms the expected specificity of the opposing interaction of THPO and LNK.

These studies demonstrate, for the first time, the importance of interaction between positive and negative regulators of cytokine signaling in controlling HSC expansion. The findings demonstrate that THPO, under physiological conditions in steady-state adult hematopoiesis, not only promotes HSC survival as previously suggested, but also stimulates HSC expansion, the extent of which is tightly restricted through the ability of LNK to negatively regulate THPO signaling. The possibility of specifically targeting these potent stimulatory and inhibitory pathways for HSC expansion should trigger renewed interest and efforts toward exploitation of cytokine pathways to promote ex vivo HSC expansion for therapeutic purposes.

\section{Materials and methods}

Mice

The $\mathrm{Lnk}^{-/-}$mouse strain was generated as previously described (Takaki et al. 2000), and the Thpo-/- strain was generated by Dr. F. de Sauvage at Genentech, Inc. (San Francisco, CA) (de Sauvage et al. 1996). Both strains were backcrossed to $\mathrm{C} 57 \mathrm{Bl} / 6$ mice for $>10$ generations. $\mathrm{Lnk}^{-/-}$mice were mated to $\mathrm{Thpo}^{-/-}$to generate $\mathrm{Lnk}^{-/-} \mathrm{Thpo}^{-/-}$mice. C57Bl/6 wild-type mice were used as controls. All animal protocols were approved by the local ethics committee at Lund University.

Isolation of hematopoietic cells and fluorescence-activated cell sorting (FACS) analysis of LIN ${ }^{-1 / o}$ SCA1 $1^{+}$KIT $T^{+}$(LSK) subpopulations

BM cells were collected from femurs and tibias by crushing in a mortar. FLs were obtained from time-matched pregnant wild-type and $\mathrm{Lnk}^{-/}$ mice at 14.5 dpc. Modifications of previously described procedures (Adolfsson et al. 2005; Yang et al. 2005) were used to evaluate the dis- tribution of cells within the LSK compartment. For more detailed information, see the Supplemental Material.

Competitive repopulation assay

The competitive repopulation assay using the congenic CD45.1/CD45.2 mouse model has been described previously (Szilvassy et al. 1990; Yang et al. 2005). Briefly, CD45.2 donor FL or BM cells were injected intravenously along with 200,000 competitor CD45.1 BM cells into lethally irradiated (900-975 cGy) CD45.1 hosts. Transplant doses (ranging from 500 to $10^{6}$ cells) were approximated to contain a limiting repopulating cell dose. PB was collected 16 wk after transplantation and analyzed for donor reconstitution by FACS. Positively reconstituted mice were defined as having a minimum of $0.1 \% \mathrm{CD} 45.2^{+}$total cells and $0.02 \%$ $\mathrm{CD} 45.2^{+}$cells of each of the myeloid $\left(\mathrm{Mac}^{-1^{+}}\right), \mathrm{B}\left(\mathrm{B} 22 \mathrm{O}^{+}\right)$, and $\mathrm{T}(\mathrm{CD} 4 /$ $\mathrm{CD}^{+}$) lineages, and CRU frequencies in the test BM sample were calculated using Limit Dilution Analysis software (StemCell Technologies, Inc.). For more detailed information, see the Supplemental Material.

In vitro clonogenic progenitor assays

Single wild-type or Lnk $^{-1-}$ LSKCD34-FLT3- $^{-}$LSKCD34+FLT3-, or LSKCD $34^{+} \mathrm{FLT} 3^{\text {hi }} \mathrm{BM}$ cells were seeded into individual wells of Terasaki plates in $20 \mu \mathrm{L}$ serum-free medium (X-vivo15; BioWhittaker), supplemented with $1 \%$ bovine serum albumin (StemCell Technologies, Inc.) and $50 \mathrm{ng} / \mathrm{mL}$ human THPO for $10 \mathrm{~d}$, at which time wells were scored for clonal growth as previously described (Adolfsson et al. 2005). CFU-GM, BFU-E, and CFU-Mk in unfractionated BM cells were evaluated in methylcellulose cultures as previously described (Adolfsson et al. 2005). For more detailed information, see the Supplemental Material.

\section{Q-PCR}

Two thousand LSKCD34-FLT3- ${ }^{-}$, LSKCD34 ${ }^{+} \mathrm{FLT3}^{-}$, and LSKCD34 ${ }^{+}$FLT3 $3^{\text {hi }}$ BM cells from 12-wk-old wild-type or $\mathrm{Lnk}^{-/-}$mice were subjected to Q-PCR analysis as recently described (Adolfsson et al. 2005). Assays-onDemand probes (InVitrogen) used were HPRT Mm00446968_m1, Lnk Mm00493156_ml, Mpl Mm00440310_ml, Hoxb4 Mm00657964_ml, and Bmi-1 Mm00776122_gH. All experiments were performed in triplicate and differences in cDNA input were compensated by normalization against HPRT expression levels.

\section{CFU-S}

BM cells (range 50,000-150,000) from adult wild-type, $\mathrm{Lnk}^{-/-}$, Thpo- ${ }^{-/-}$, or $\mathrm{Lnk}^{-/-} \mathrm{Thpo}^{-/-}$mice were transplanted into lethally irradiated wild-type recipients, and $12 \mathrm{~d}$ after transplantation macroscopic colonies were evaluated after fixation of spleens in Tellesnicky's fixative, as previously described (Yang et al. 2005).

Peripheral blood cell analysis

PB was collected from the retro-orbital sinus into EDTA-coated tubes (Sarstedt) and analyzed in an automated cell counter (Sysmex). Differential counts were also obtained by microscopic examination of May-Grünwald/Giemsa-stained PB smears. 
Statistics

Unless otherwise stated, the statistical significance of differences between wild-type, $\mathrm{Lnk}^{-/-}$, $\mathrm{Thpo}^{-/-}$, and $\mathrm{Lnk}^{-/-} \mathrm{Thpo}^{-/-}$mice was determined using the two-tailed Mann-Whitney test.

\section{Acknowledgments}

We thank Dr. John Adamson for providing the Thpo-/- mice. The expert technical assistance of Anna Fossum, Zhi Ma, Lilian Wittman, Ingbritt Åstrand-Grundström, Marie Magnusson, Therese Blidberg, Jalal Taneera, Stuart Walsh, and Gunilla Gärdebring is highly appreciated. We also thank Dr. Warren Alexander for critical review of the manuscript. These studies were supported by grants from ALF (Government Public Health Grant), Alfred Österlund Foundation, the Swedish Research Council, the Swedish Foundation for Strategic Research (SSF), the Swedish Cancer Society, and the EU project LHSB-CT-2003-503005 (EuroStemCell). J.A. is supported by a fellowship from the Leukemia and Lymphoma Society. The Lund Stem Cell Center is supported by a Center of Excellence grant from SSF.

\section{References}

Adolfsson, J., Borge, O.J., Bryder, D., Theilgaard-Monch, K., AstrandGrundstrom, I., Sitnicka, E., Sasaki, Y., and Jacobsen, S.E. 2001. Upregulation of Flt3 expression within the bone marrow $\mathrm{Lin}^{-} \mathrm{Scal}^{+} \mathrm{c}-\mathrm{kit}{ }^{+}$ stem cell compartment is accompanied by loss of self-renewal capacity. Immunity 15: 659-669.

Adolfsson, J., Mansson, R., Buza-Vidas, N., Hultquist, A., Liuba, K., Jensen, C.T., Bryder, D., Yang, L., Borge, O.J., Thoren, L.A., et al. 2005. Identification of $\mathrm{Flt}^{+}$lympho-myeloid stem cells lacking erythro-megakaryocytic potential a revised road map for adult blood lineage commitment. Cell 121: 295-306.

Antonchuk, J., Sauvageau, G., and Humphries, R.K. 2002. HOXB4-induced expansion of adult hematopoietic stem cells ex vivo. Cell 109: 39-45.

Borge, O.J., Ramsfjell, V., Veiby, O.P., Murphy Jr., M.J., Lok, S., and Jacobsen, S.E. 1996. Thrombopoietin, but not erythropoietin promotes viability and inhibits apoptosis of multipotent murine hematopoietic progenitor cells in vitro. Blood 88: 2859-2870.

Carver-Moore, K., Broxmeyer, H.E., Luoh, S.M., Cooper, S., Peng, J., Burstein, S.A., Moore, M.W., and de Sauvage, F.J. 1996. Low levels of erythroid and myeloid progenitors in thrombopoietin-and c-mpl-deficient mice. Blood 88: 803-808.

Christensen, J.L. and Weissman, I.L. 2001. Flk-2 is a marker in hematopoietic stem cell differentiation: A simple method to isolate longterm stem cells. Proc. Nat1. Acad. Sci. 98: 14541-14546.

de Sauvage, F.J., Carver-Moore, K., Luoh, S.M., Ryan, A., Dowd, M., Eaton, D.L., and Moore, M.W. 1996. Physiological regulation of early and late stages of megakaryocytopoiesis by thrombopoietin. I. Exp. Med. 183: 651-656.

Ema, H., Sudo, K., Seita, J., Matsubara, A., Morita, Y., Osawa, M., Takatsu, K., Takaki, S., and Nakauchi, H. 2005. Quantification of self-renewal capacity in single hematopoietic stem cells from normal and Lnk-deficient mice. Dev. Cell 8: 907-914.

Iscove, N.N. and Nawa, K. 1997. Hematopoietic stem cells expand during serial transplantation in vivo without apparent exhaustion. Curr. Biol. 7: 805-808.

Ito, T., Tajima, F., and Ogawa, M. 2000. Developmental changes of CD34 expression by murine hematopoietic stem cells. Exp. Hematol. 28: 1269-1273.

Iwama, A., Oguro, H., Negishi, M., Kato, Y., Morita, Y., Tsukui, H., Ema, H., Kamijo, T., Katoh-Fukui, Y., Koseki, H., et al. 2004. Enhanced self-renewal of hematopoietic stem cells mediated by the polycomb gene product Bmi-1. Immunity 21: 843-851.

Jacobsen, S.E. 2005. Defining 'stemness': Notch and Wnt join forces? Nat. Immunol. 6: 234-236.

Kimura, S., Roberts, A.W., Metcalf, D., and Alexander, W.S. 1998. Hematopoietic stem cell deficiencies in mice lacking c-Mpl, the receptor for thrombopoietin. Proc. Nat1. Acad. Sci. 95: 1195-1200.

Miller, C.L. and Eaves, C.J. 1997. Expansion in vitro of adult murine hematopoietic stem cells with transplantable lympho-myeloid reconstituting ability. Proc. Natl. Acad. Sci. 94: 13648-13653.

Miller, C.L., Rebel, V.I., Lemieux, M.E., Helgason, C.D., Lansdorp, P.M., and Eaves, C.J. 1996. Studies of W mutant mice provide evidence for alternate mechanisms capable of activating hematopoietic stem cells. Exp. Hematol. 24: 185-194.

Molofsky, A.V., Pardal, R., Iwashita, T., Park, I.K., Clarke, M.F., and Morrison, S.J. 2003. Bmi-1 dependence distinguishes neural stem cell self-renewal from progenitor proliferation. Nature 425: 962-967.

Morrison, S.J., Wandycz, A.M., Akashi, K., Globerson, A., and Weissman, I.L. 1996. The aging of hematopoietic stem cells. Nat. Med. 2: 10111016.

Ogawa, M. 1993. Differentiation and proliferation of hematopoietic stem cells. Blood 81: 2844-2853.

Pawliuk, R., Eaves, C., and Humphries, R.K. 1996. Evidence of both ontogeny and transplant dose-regulated expansion of hematopoietic stem cells in vivo. Blood 88: 2852-2858.

Pestina, T.I., Cleveland, J.L., Yang, C., Zambetti, G.P., and Jackson, C.W. 2001. Mpl ligand prevents lethal myelosuppression by inhibiting p53dependent apoptosis. Blood 98: 2084-2090.

Reya, T., Morrison, S.J., Clarke, M.F., and Weissman, I.L. 2001. Stem cells, cancer, and cancer stem cells. Nature 414: 105-111.

Sauvageau, G., Iscove, N.N., and Humphries, R.K. 2004. In vitro and in vivo expansion of hematopoietic stem cells. Oncogene 23: 7223 7232.

Solar, G.P., Kerr, W.G., Zeigler, F.C., Hess, D., Donahue, C., de Sauvage, F.J., and Eaton, D.L. 1998. Role of c-mpl in early hematopoiesis. Blood 92: 4-10.

Sorrentino, B.P. 2004. Clinical strategies for expansion of haematopoietic stem cells. Nat. Rev. Immunol. 4: 878-888.

Szilvassy, S.J., Humphries, R.K., Lansdorp, P.M., Eaves, A.C., and Eaves, C.J. 1990. Quantitative assay for totipotent reconstituting hematopoietic stem cells by a competitive repopulation strategy. Proc. Nat1. Acad. Sci. 87: 8736-8740.

Takaki, S., Sauer, K., Iritani, B.M., Chien, S., Ebihara, Y., Tsuji, K., Takatsu, K., and Perlmutter, R.M. 2000. Control of B cell production by the adaptor protein lnk. Definition of a conserved family of signalmodulating proteins. Immunity 13: 599-609.

Takaki, S., Morita, H., Tezuka, Y., and Takatsu, K. 2002. Enhanced hematopoiesis by hematopoietic progenitor cells lacking intracellular adaptor protein, Lnk. I. Exp. Med. 195: 151-160.

Till, J.E. and McCulloch, E.A. 1961. A direct measurement of the radiation sensitivity of normal mouse bone marrow cells. Radiat. Res. 14: $213-222$.

Tong, W. and Lodish, H.F. 2004. Lnk inhibits Tpo-mpl signaling and Tpo-mediated megakaryocytopoiesis. J. Exp. Med. 200: 569-580.

Tong, W., Zhang, J., and Lodish, H.F. 2005. Lnk inhibits erythropoiesis and Epo-dependent JAK2 activation and downstream signaling pathways. Blood 105: 4604-4612.

Velazquez, L., Cheng, A.M., Fleming, H.E., Furlonger, C., Vesely, S., Bernstein, A., Paige, C.J., and Pawson, T. 2002. Cytokine signaling and hematopoietic homeostasis are disrupted in Lnk-deficient mice. J. Exp. Med. 195: 1599-1611.

Wilson, A., Murphy, M.J., Oskarsson, T., Kaloulis, K., Bettess, M.D., Oser, G.M., Pasche, A.C., Knabenhans, C., Macdonald, H.R., and Trumpp, A. 2004. c-Myc controls the balance between hematopoietic stem cell self-renewal and differentiation. Genes \& Dev. 18: $2747-$ 2763.

Yang, L., Bryder, D., Adolfsson, J., Nygren, J., Mansson, R., Sigvardsson, M., and Jacobsen, S.E. 2005. Identification of $\mathrm{Lin}^{-} \mathrm{Sca} 1^{+} \mathrm{kit}^{+} \mathrm{CD} 34^{+} \mathrm{Flt} 3^{-}$ short-term hematopoietic stem cells capable of rapidly reconstituting and rescuing myeloablated transplant recipients. Blood 105: 27172723.

Yokouchi, M., Suzuki, R., Masuhara, M., Komiya, S., Inoue, A., and Yoshimura, A. 1997. Cloning and characterization of APS, an adaptor molecule containing $\mathrm{PH}$ and $\mathrm{SH} 2$ domains that is tyrosine phosphorylated upon B-cell receptor stimulation. Oncogene 15: 7-15.

Zhang, C.C. and Lodish, H.F. 2005. Murine hematopoietic stem cells change their surface phenotype during ex vivo expansion. Blood 105: 4314-4320.

Zhang, P., Iwasaki-Arai, J., Iwasaki, H., Fenyus, M.L., Dayaram, T., Owens, B.M., Shigematsu, H., Levantini, E., Huettner, C.S., Lekstrom-Himes, J.A., et al. 2004. Enhancement of hematopoietic stem cell repopulating capacity and self-renewal in the absence of the transcription factor C/EBP $\alpha$. Immunity 21: 853-863. 


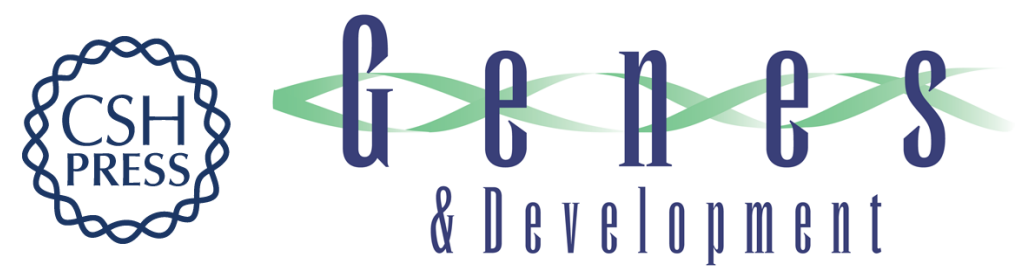

\section{Cytokines regulate postnatal hematopoietic stem cell expansion: opposing roles of thrombopoietin and LNK}

Natalija Buza-Vidas, Jennifer Antonchuk, Hong Qian, et al.

Genes Dev. 2006, 20:

Access the most recent version at doi:10.1101/gad.385606

Supplemental http://genesdev.cshlp.org/content/suppl/2006/08/04/20.15.2018.DC1
Material

References This article cites 36 articles, 18 of which can be accessed free at:

http://genesdev.cshlp.org/content/20/15/2018.full.html\#ref-list-1

License

Email Alerting Receive free email alerts when new articles cite this article - sign up in the box at the top

Service

right corner of the article or click here.

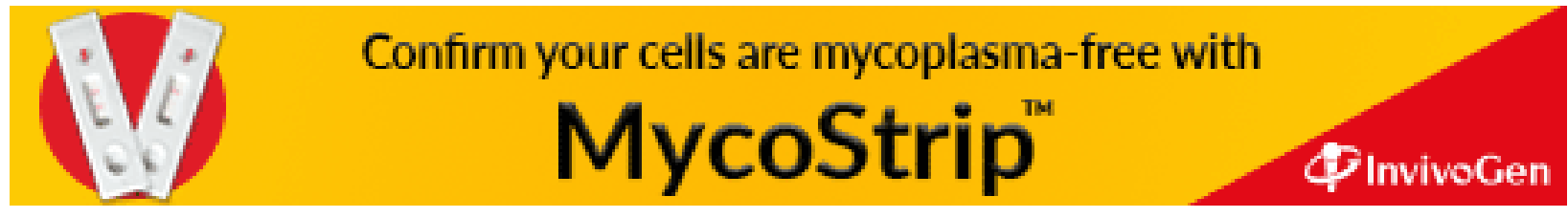

